\title{
Certain Investigations on Bank Customer and Relationship Improvement using Machine Learning
}

\author{
J Shiny Duela ${ }^{1}$, Dioline Sara ${ }^{2}$
}

\{shiny.duela@gmail.com ${ }^{1}$,dsara@gitam.edu ${ }^{2}$ \}

Department of Computer Science \& Engineering, SRM Institute of Science and

Technology,Ramapuram Campus, Chennai ${ }^{1}$, Department of Electronics \& Communication Engineering, Gitam School of Technology, Bangalore ${ }^{2}$

\begin{abstract}
Visual information investigation includes utilizing present-day P.C. illustrations and show instruments to investigate information. The utilization of such visual research techniques has gotten progressively across the board all through the sociologies. This proposed framework centres around client information in business banks and expects to utilize visual portrayals and connection strategies to give another vision of client relationship the board or customer relationship management (CRM).Features of the discoveries incorporate that consumer loyalty has a remarkable positive impact on client dedication in the financial assistance industry, particularly with the salary improving, the connection between them turns out to be increasingly robust. At last, recommendations are advanced on the most proficient method to enhance consumer loyalty and increment client dedication. They are continually vigilant for utilizing them to improve their business advantages, for example, right item advancement to right sort of clients, typical usage of self- administration channels, diminished visits to branches for money exchanges, etc. Select Bank's analysis of bank customer awareness about who to bank financing, credit to give something constraints. In addition, it can make the guarantor to show signs of improvement in their potential and existing customers to understand. As customers analyze is significantly essential for such activities, analysis of a client to create a clear framework for banks. The central aspect of this study is to use the used dataset (labelled) and to create a new label as the target for classification, which reduces the clustering execution time and gets the best accuracy results. The data set ('default of credit card clients) is obtained from the archive of UCI (University of California, Irvine) M.L. Repository.
\end{abstract}

Keywords: Unified Analysis, Machine Learning, CRM, Clustering Algorithm, DBSCAN 


\section{Introduction}

In the budget line area, the bank has included information about the customer and their communication history huge data sets. It is so, banks need these huge data integration package called group of small parts have to check these customers an alternative approach, using it in a perfect way an appropriate proposed procedures to achieve the most critical benefits, profitability, customer reliability growth. Customer analysis or customer frequency. Help analyze customer profiles, which provides continuous money depends on a lot of features [1] their clients a complete description of age. Customer department intends to create dependent on any particular quality customer gatherings (such as language environment, communication, age, phone, pay segment division), or their behavior (social division of labor). In any case, "client division and profiling" is considered to be practically mirror images of each other. Banks also face various troubles like the default graphics / expectations, Customer maintenance, customer analysis and risk executives behind achieve higher profitability and reduce the hazards of different motives. Therefore, the most fundamental is well separated from the customer, relaxation such trouble. AI show P.C. the most effective way to confirm how to find not just work on the model, the work is there, but then who and cloud conditions and limitations. The information index ( customer visas default) obtained by the library M.L. chronicle UCI (UC Irvine) is.

AI not only help in updating affiliation levels with current customers [2], be that as it may, it moreover accept a noteworthy activity in anticipating the lead of customers reliant on a particular get-together of occasions or models distinguishes their future methodology, anticipating offering focused on layaway items and proposals to the clients and henceforth improving client relationship the board (CRM). At the point when client picks up benefit, it expands CRM and by implication benefit banks. It moved the focus to the customer and changed the imagined by banks in their current game plan. The Density-based grouping AI method is utilized in this research, and their applications are applied to a genuine dataset from UCI (the University of California, Irvine) Machine Learning Repository, and afterward contrast the precision proportion and the current framework. The pre-owned AI strategies are tied in with profiling the client practices utilizing exchange/Financial information just utilizing K-mean Clustering Algorithm. Whatever residual pieces of this paper is filtered through as follows: In Section II Literature Survey are presented; in Section III, we examined System Architecture and work process diagram and talked about concerning issue definition, the entire structure and rule checks related with this paper. In Section IV, we share Result gained with the dataset to the extent visual depiction. Assessment with outcomes dependent on estimation are presented in Section V.

\section{Literature Survey}

There have been numerous investigations completed on virtual financial reception, yet considers did in Malaysia to upgrade the virtual economic system selection are restricted. Along these lines, the purpose of this survey is to apply the model to guide a virtual financial technology and the use of technology acceptance model (TAM), found that a key factor in the firm's virtual 
discerning customers complete financial procedures in Malaysia. Theory of Reasoned Action (TRA) and Diffusion of Innovation (DOl) speculations to embrace computer-generated financial methodologies to address the client's issue with the progression in instalment framework and innovation. From the preliminary study results show that the factors now test the free is worth noting that the impact of the measurement device is suitable for this test, a nearby bank financial technology in Malaysia and dedication to customer relationship tests as recommended by the instrument. This exploration can be helpful for future scientists right now space. In any case, a result may contrast with a more significant example size led to this examination, the main drawback of the system is that virtual banking strategy adoption is limited in it [1].

As per the information highlight of the client's utilization records of bank POS machine and the examination relying upon the absolute prerequisites another displaying structure on utilization conduct of the bank, POS machines are introduced right now, further research on the usage strategy for primary angles in the model is done. Initially, we direct information discretization and client division by K-mean calculation and Kohonen organize bunching calculation separately, break down and think about the outcomes thoroughly, and eventually get the ideal aftereffect of removing great clients. At that point during the time spent mining the top-notch client's utilization trademark, we utilize the C5.0 calculation to break down the trial and assess the trial results after getting the great client's utilization records through client division results. At last, we get the information base of great buyer's utilization conduct which can offer help to banks and shippers to settle on the right choice. Its major drawback is that its cost is very high [2].The view of Internet banking was secure and valuable and high on protection. In the interim, the impression of SMS banking was anything but difficult to get to and high on security. Moreover, telephone banking was seen to be the most costly and off base. Future examinations are required, notwithstanding utilizing other multivariate procedures that would likewise have the option to include different properties that are progressively compelling. High complexity and low security is the major drawback for the previous systems [3].In Thailand, it has driven more shoppers to lead money related exchanges and other financial exercises on their cell phones. In any case, buyer trust assumes a significant job in the improvement of long-haul connections in the online condition among banks and their clients. Hence, the motivation behind this exploration was to recognize how much a few impacting factors that influence trust of Thai customers in portable banking. Banks ought to offer support by feature improving security to upgrade the trust of their clients and increment the use of portable banking. Security is affecting the trust of mobile banking customers, is the most significant drawbacks [4] and one of the most critical factors. Semi-directed learning has been conclusively associated with the exploration fields of machine learning. Moreover, we improve the characterization execution of the DFCM-MC, apply the re-inspecting strategy to deal with the imbalanced information for arrangement. The trial results exhibit that our proposed approach performs better because of their ability to perceive and merge primary data from unaided information. This technique can affect performance prediction model, and also the data into low-dimensional learning efficiency, but to establish a significant disadvantage of this system, and can be solved by DBSCAN [5]. The framework can dispose of disdainful and uninterested qualities and select the helpful ones. It can likewise misuse unpretentious nonlinear connection between characteristics [6]. So as to show the adequacy of the structure, the authors have utilized a few freely accessible programming flaw informational indexes and contrasted the presentation of our technique and that of some current strategies. For assessing the element standardization methods, backengendering counterfeit neural system [BPANN] and bolster vector machine [SVM] classifier models are utilized[7]. Results show that that standardization of highlights has critical impact on the arrangement exactness. General Terms Pattern Recognition, Medical Image Processing. 
[8] demonstrates that LightGBM or Xgboost has a decent presentation in the expectation of clear-cut reaction factors and has a decent application esteem in the huge information period. The examination of models with boosting and smoothing shows that blunder rate is preferred measurement over region under bend (AUC) proportion. It is presumed that counterfeit neural system (ANN) is preferred option over choice tree and calculated relapse when information accessibility is high in dataset[9].

\section{System Architecture}

The framework in figure 1 represents the overall transfer of information from fetching the data for the process of data normalization makes data dimensionless. It is helpful for characterizing standard files. After normalization, all information on the area and size of the first data might be lost. It is important to normalize factors in situations where the uniqueness measure, for example, the Euclidean separation, is delicate to the distinctions in the extents or sizes of the info factors. The methodologies of normalization of factors are basically of two kinds: worldwide normalization and inside bunch normalization.and the technique followed for client profiling can be handily utilized by any bank, as the phrasings, strategic approaches, rules, database fields, exchange types, business destinations insufficient utilization of innovation and so forth are nearly the equivalent for all the Indian open segment banks As the motivation behind this activity was to recognize client bunches that have the common behavioral example, it was important to discover for every client, the tasks he/she is doing and the aggregate sum associated with the separate assistance.

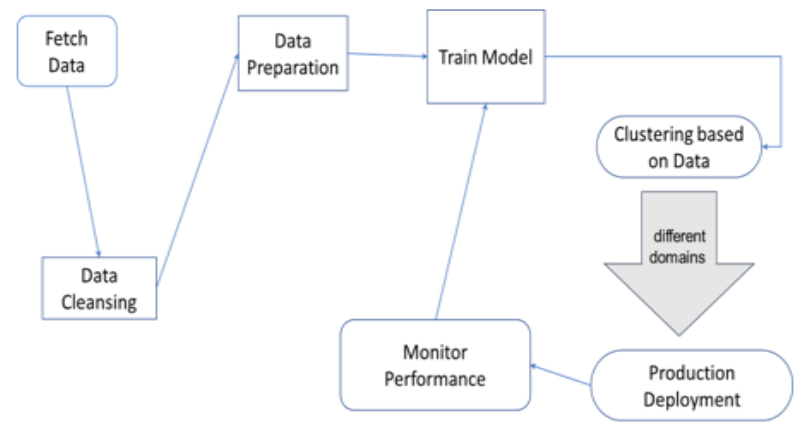

Fig. 1. System Architecture

The potential estimation of the customers is to highlight the client for any of similar sides of business inside the region of the endeavor might be the advantages. This worth is free of any outside the venture, for any organizations are no distinctions. At the point when undertaking and client exchange, the venture through the endeavors to put some portion of the potential client esteem into genuine value. The advantages of this proposed system are that it does not require a-prior specification of several clusters, it can identify noise data while clustering, and DBSCAN algorithm can find arbitrarily size and arbitrarily shaped clusters. 


\subsection{Data Assessment}

Modeling exploratory data analysis (EDA) explained largely dependent on the visualization and graphic data. Although statistical modeling provides a relationship between the variables of a simple low-dimensional representation, they generally require analytical techniques and knowledge of advanced mathematical principles.

\subsection{Preprocessing}

Traditional text preprocessing is an important symbol has three main components, standardization, and noise removal. About symbolic text string into smaller pieces, or "token." Paragraphs and sentences can be the symbol into symbolic language sentence can be expressed. The goal of the standardization of fair competition on all the books, for example, to convert all characters to lowercase. Noise removal to clean up the text, for example, remove the extra space. It becomes the token list for further processing, such as parsing input or text mining. Tokenized main purpose is to identify the necessary keywords. Inconsistency may be a different number and time format.

\subsection{Feature Engineering}

Highlight determination techniques can be recognized into three classes: channels, coverings, and inserted/mixture strategy. Coverings strategies perform well than channel techniques in light of the fact that the component determination process is streamlined for the classifier to be utilized. Channel strategies have low computational expense and quicker yet with wasteful unwavering quality in arrangement when contrasted with covering techniques and better appropriate for high dimensional informational collections. A cross breed approach utilizes both an autonomous test and execution assessment capacity of the element subset.

Fuzzy feature selection metric (FFSM) will be assigned a higher score to a class which function only occurs continuously. FFSM score calculation for each element. This method assignee scores close to 1 , if the function is clear; otherwise, it is assigned with a score close to zero based on a given threshold value and the threshold value, having a score below the threshold FFSM characteristic and a filter having a rating FFSM. It is higher than the threshold value for the element in the learning phase.

\subsection{Prediction}

DBSCAN is commonly used in data mining and machine learning because it provides a common use in many technological advances in the present case the algorithm clustering algorithm. Using a set of points, DBSCAN groups together is based on the Euclidean distance clusters close to each other. This also marks a point in the low-density region. Association forecasts that and structure, the data hard to find, but can come in patterns and predict trends related discovered in handy. And thus, this way, the prediction algorithm works in the advancement of the current process. DBSCAN Clusters are numerous areas in the information space, isolated by districts of the lower density of focuses.

Parcelling techniques (K-means, PAM clustering) and various levelled clustering work for finding round moulded clusters or arched clusters depicted in figure 2. They are appropriate just for smaller and all-around isolated groups. Additionally, they are likewise seriously influenced by the nearness of commotion and exceptions in the information. Genuine information may contain inconsistencies- 
i) Clusters can be of a subjective shape; for example, those appeared in the figure underneath. ii)Data may include commotion.

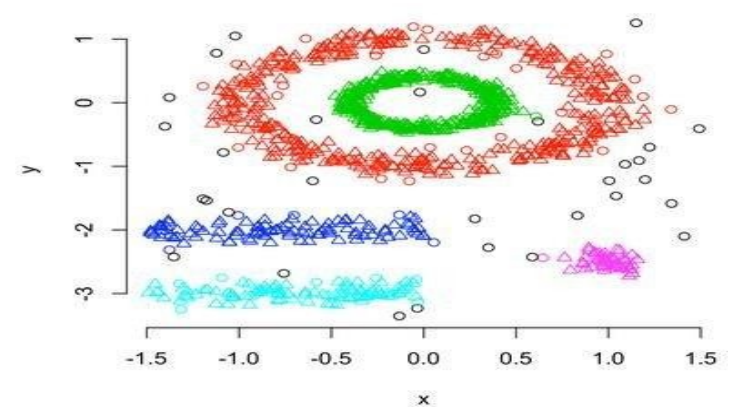

Fig 2. Density-based clustering

\section{Results and Discussion}

The dataset obtained from the University of California machine learning repository is depicted in Figure 3.

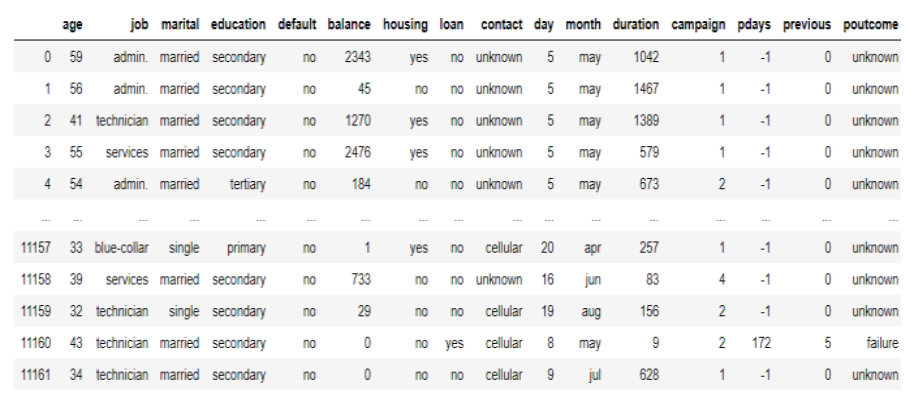

11162 rows $\times 16$ column

Fig 3 Dataset obtained from UCI M.L. repository

In the further process, these datasets are used to extract all the essential information and made clusters according to the need and are prepared based on comparing different aspect such that useful information/data to get removed from the dataset. Once, all the data is gathered as in figure 4 , it then compared based on different characteristics such that important information gets extracted, and the model is hence trained for further process shown in figure 5,6,7,8. 

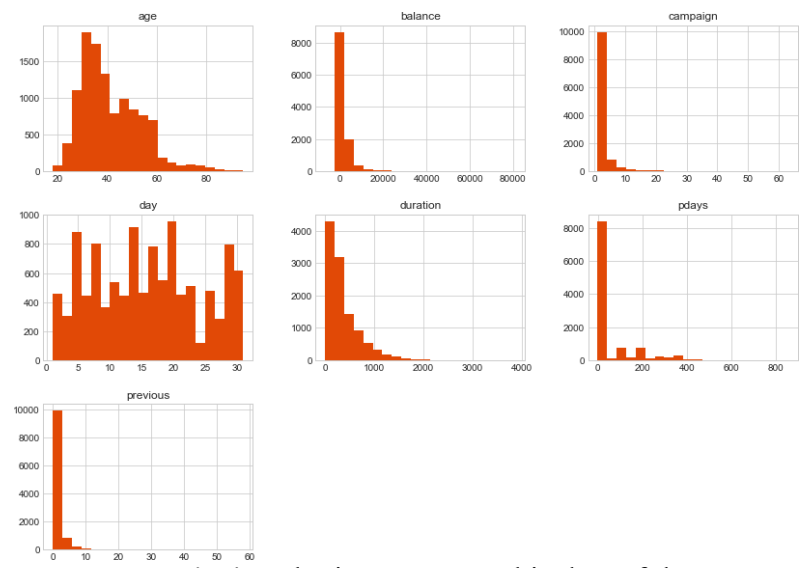

Fig 4 Gathering Demographic data of the customer
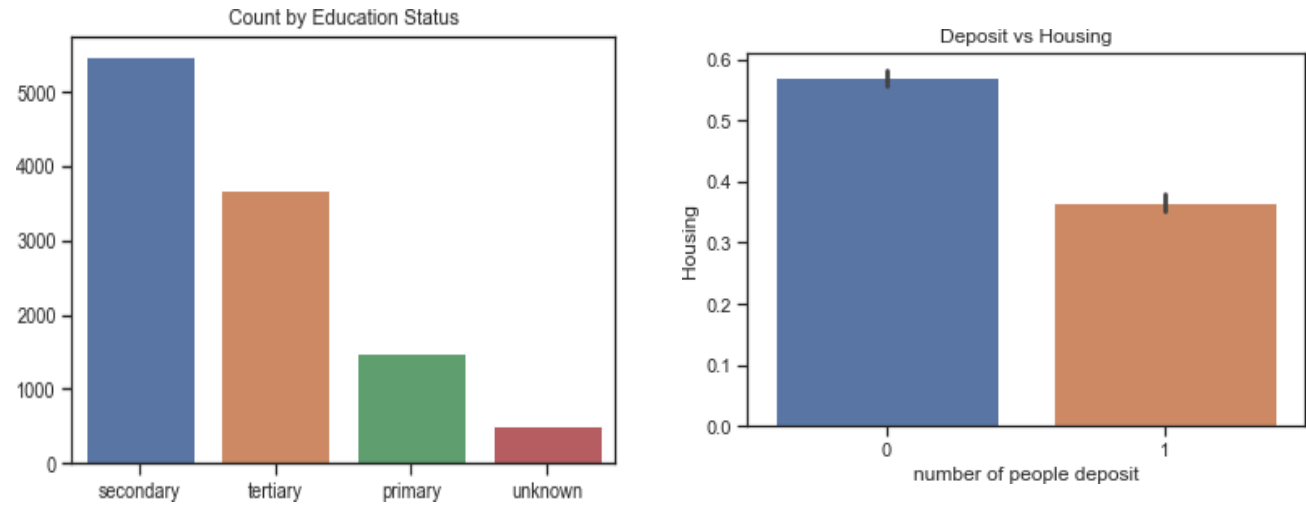

Fig 5 Comparing deposits and Loans from Dataset

Fig 6 Comparing deposits and Housing from Dataset
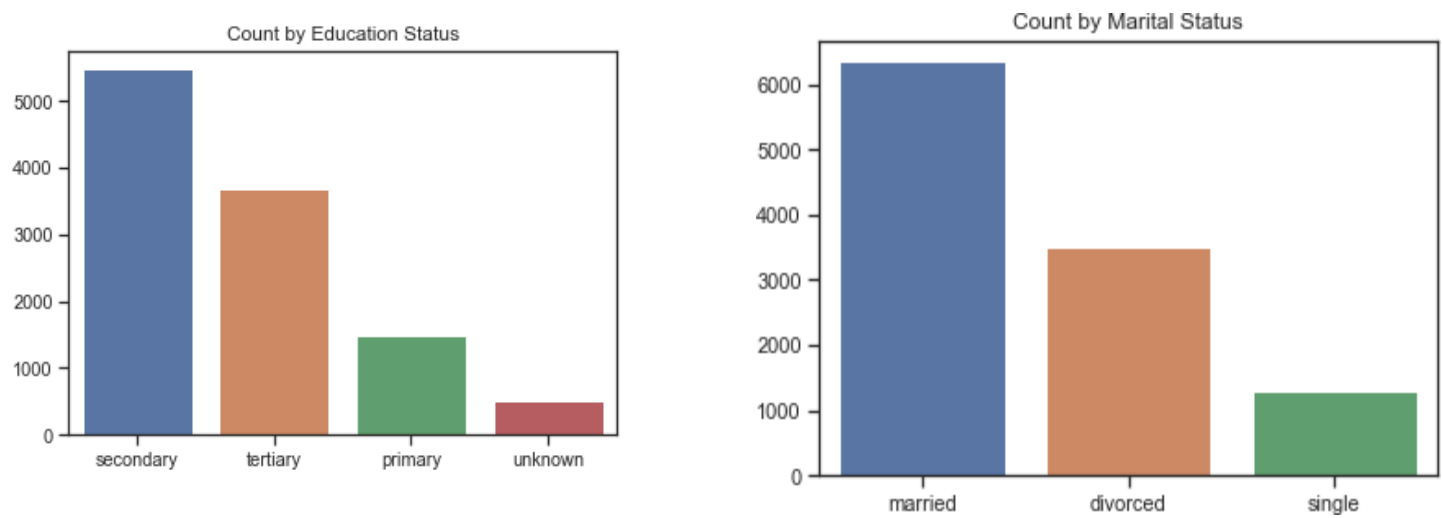

Fig 7 Representing the education status of customers Fig 8 Representing the Marital Status of customers

Different customers are compared based on the deposits and the housing loans or are compared based on deposits, or outstanding loan is checked based on the data provided. Once, the 
information is gathered, it then compared based on the requirement to extract maximum out of it to obtain and train the model shown in figure 9

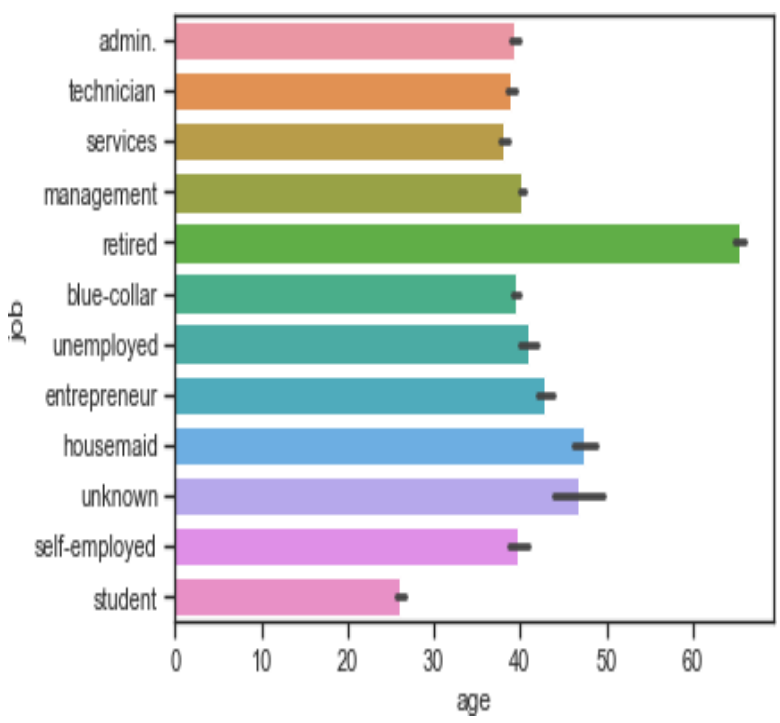

Fig 9 Comparing Different profession with age

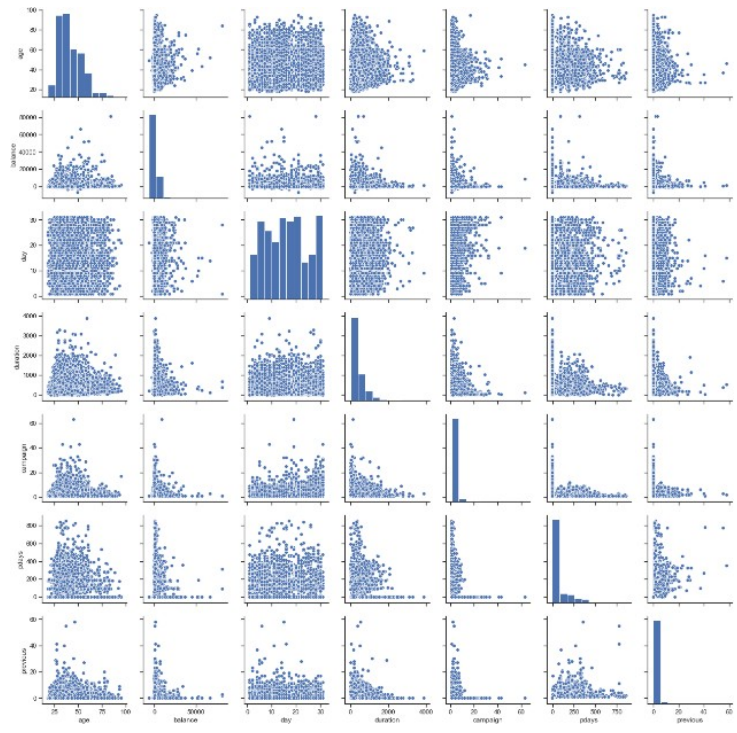

Fig 10 Graph showing density-based clustering

After comparing the desired characteristics, the obtained data is then used to compare and contrast various factors to create distinct density-based clusters such that denser areas in the graph show identical values and less dense regions generally doesn't form a group or are not of the same type. Thus, the last result in figure 10 shows the density-based clustering, which is used to classify customers.
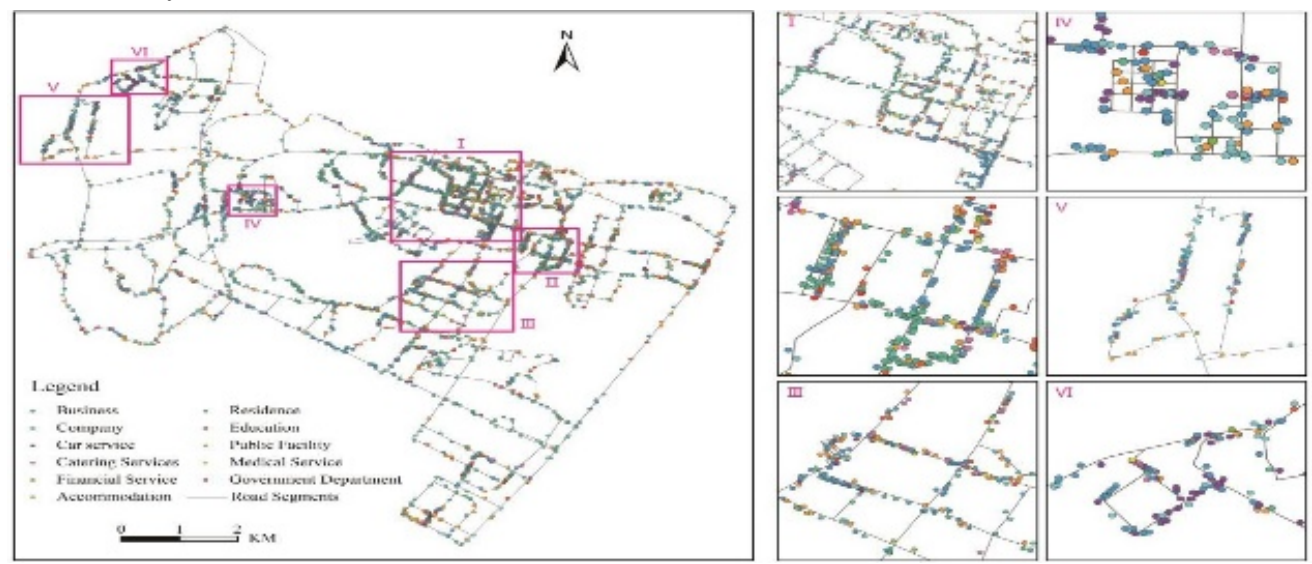

Fig 11 Highlights of all clusters formed in an area 
In spite of the fact that the MLF technique has a higher precision than other FMM strategies, as appeared in figure 11 and 12, DBSCAN's runtime is as short as those of the other K-means strategies. Additionally this technique has typical number of proper density clusters in cases where the training tests do not have wide covered area.

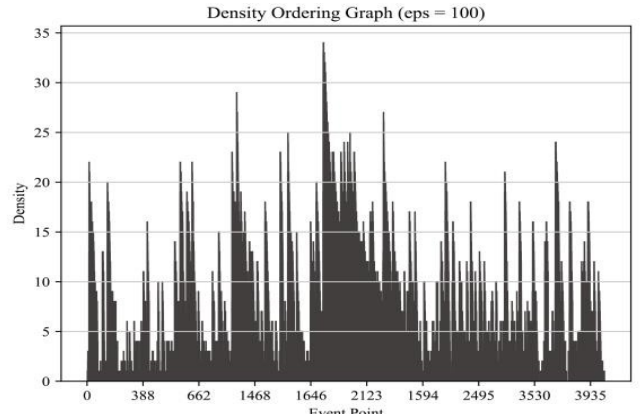

(a)

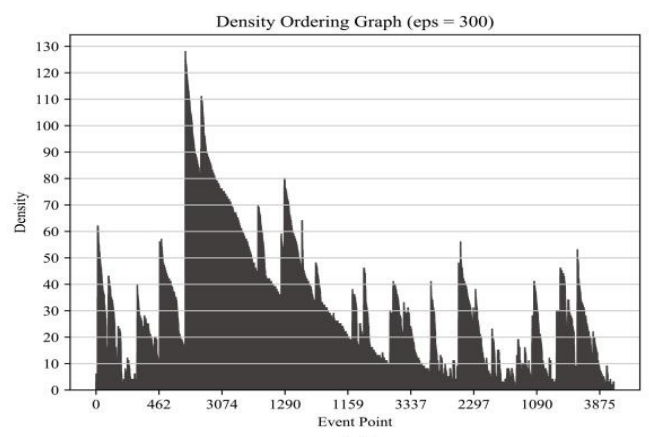

(c)

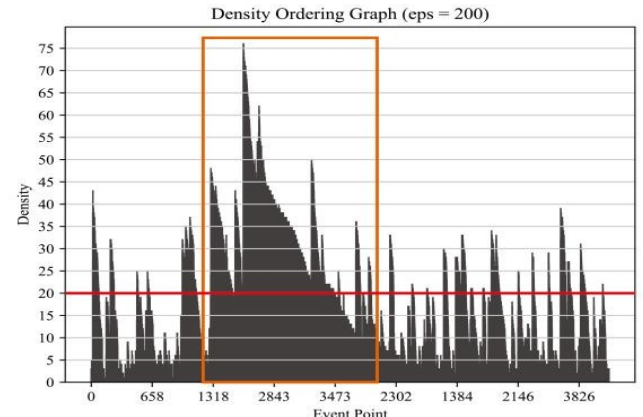

(b)

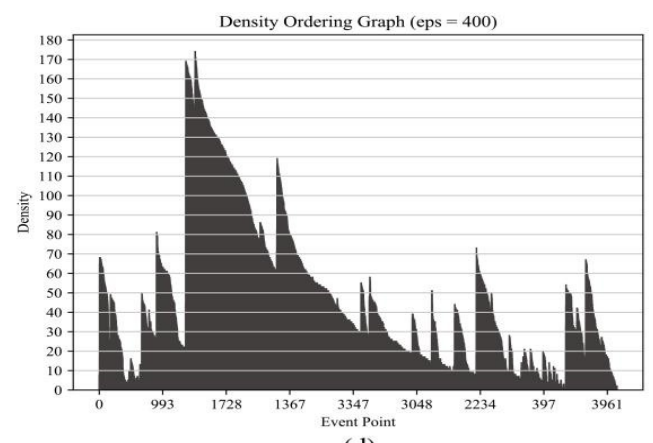

(d)

Fig 12 Density representation of different customers based on demographic data

A result assessment on the equal dataset is in like manner uncovered in the outcome. This investigation stretched out the DBSCAN calculation to arrange occasions and proposed another grouping calculation named DBSCAN. Profiling helps in the age of customer accounts, which allows the keeps money with a full portrayal of their clients enables a huge amount of consumer details. Client areas intends to portray the gatherings of clients dependent on either specific qualities (for example Area, exchange, tenure, calling, salary for partly allowance division) or their conduct (for overall distribition). Nevertheless, 'client's division and profiling' are considered as practically a mirror image of one another. Banks are confronting various troubles like default conjecture/expectation, customer maintenance, hazard the board, and customer profiling for different reasons for achieving higher profitability and reducing the hazard. 


\section{Conclusion}

In this work, how a bank's office level exchange information of clients can be utilized for client profiling is introduced. The system proposed to achieve the equivalent is clarified, and all the phases in the profiling exercise are represented through the fitting middle outcomes. The deciphered outcomes unmistakably demonstrated how the bank could viably utilize them in accomplishing its business destinations. It is to be noticed that in the present setting of innovation acceptance, this work is noteworthy for the Indian banks as branches are the main client touchpoints and business advancing focuses. The work can additionally be upgraded when banks can catch client's segment data.

With the use of DBSCAN over the other k-mean and improved k-mean algorithm, it has given the system the more efficient efficiency compared to the others and which would provide the better customer relationship management and also with better handling of the data within the system and working upon them.

\section{References}

[1] Conference proceedings paper: Tham, Jacqueline \& Khin, Aye. A Conceptual Model of Virtual Banking Strategies in Achieving Customers, International Conference on Information and Communication Technology (ICICTM),2016,pp:178-182

[2] Conference proceedings paper: Xie, Yonghong \& Zhang, Dezheng \& Fu, Yajing \& Li, Xiaohui \& Li, Hui.Applied research on customer's consumption behavior of bank POS machine based on data mining,IEEE 9th Conference on Industrial Electronics and Applications (ICIEA), 2014,pp:1975-1979.

[3] Conference proceedings paper: R. R. W. Giri, B. R. Santoso, A. Mustikasari and M. K. Bratawisnu. Consumer perceptual mapping towards e-banking channel: (A study of bank BRI customer in Indonesia);5th International Conference on Information and Communication Technology (ICoIC7), Malacca City, 2017,pp. 1-4

[4] Journal Article: Sabah Abdullah Al-Somali, Roya Gholami, Ben Clegg. An investigation into the acceptance of online banking in Saudi Arabia.Technovation,2009,29(2),pp.130-141

[5] Journal Article: Arshad, Ali \& Riaz, Saman \& Jiao, Licheng. Semi-Supervised Deep Fuzzy CMean Clustering for Imbalanced Multi-class Classification. IEEE Access.2019, 7,pp: 28100 28112.

[6] Journal Article: P. Singh, N. R. Pal, S. Verma and O. P. Vyas.Fuzzy Rule-Based Approach for Software Fault Prediction," in IEEE Transactions on Systems, Man, and Cybernetics: Systems,47(5), 2017, pp. 826-837.

[7] Journal Article: KumarSingh, Bikesh \& Verma, Kesari \& Thoke, A. Investigations on Impact of Feature Normalization Techniques on Classifier's Performance in Breast Tumor Classification, International Journal of Computer Applications.116,2015, pp:11-15.

[8] Journal Article: Yang, Shenghui \& Zhang, Haomin. Comparison of Several Data Mining Methods in Credit Card Default Prediction, Intelligent Information Management.10.2018, pp: 115-122.

[9] Journal Article: Imtiaz, Sharjeel \& Brimicombe, Allan. A Better Comparison Summary of Credit Scoring Classification, International Journal of Advanced Computer Science and Applications, 8, 2017, pp:1-4. 
